\title{
Desigualdade racial nas boas práticas e intervenções obstétricas no parto e nascimento em maternidades da Rede Cegonha
}

\author{
Racial inequality in obstetric good practices and interventions \\ in labor and birth care in Rede Cegonha
}

\author{
Maria Teresa Seabra Soares de Britto e Alves (https://orcid.org/0000-0002-4806-7752) ${ }^{1}$ \\ Deysianne Costa das Chagas (http://orcid.org/0000-0003-0239-6662) ${ }^{1}$ \\ Alcione Miranda dos Santos (https://orcid.org/0000-0001-9711-0182) ${ }^{1}$ \\ Vanda Maria Ferreira Simões (https://orcid.org/0000-0001-8351-1348) ${ }^{1}$ \\ Barbara Vasques da Silva Ayres (https://orcid.org/0000-0002-6228-5932) ${ }^{2}$ \\ Gilmara Lúcia dos Santos (https://orcid.org/0000-0002-6116-9163) ${ }^{3}$ \\ Antônio Augusto Moura da Silva (https://orcid.org/0000-0003-4968-5138) ${ }^{1}$
}

${ }^{1}$ Centro de Ciências da Saúde, Universidade Federal do Maranhão. R. Barão de Itapary 155, Centro. 65020070 São Luís MA Brasil. mtseabra@gmail.com ${ }^{2}$ Escola Nacional de Saúde Pública Sérgio Arouca, Fiocruz. Rio de Janeiro RJ Brasil.

${ }^{3}$ Ministério da Saúde, Brasil. Brasília DF Brasil.

\begin{abstract}
This study aimed to evaluate the racial inequality on childbirth care at the Rede Cegonha (Stork Network) using obstetric good practice and interventions indicators. Racial inequality, measured by the total effect of ethnicity/skin color in the crude model, was seen in many indicators. After adjusting for mediators, such as age, schooling, parity, high-risk hospital, and geographic macro-regions, the persistent direct effect suggests racial discrimination against black women with lower partograph completion (PR 0.88; 95\% CI 0.80-0.95). Black women stayed less in lithotomy (PR 0.93; 95\% CI 0.89-0.98), performed less episiotomy (PR 0.81; 95\% CI 0.68-0.96), and had less episiotomy suturing pain (PR 0.66; 95\% CI 0.51 - 0.87) when compared to white women, suggesting more good practice applied to black women. However, according to the interventionist care model still adopted by many professionals, these practices are routine, and lower achievement in black women would be better interpreted as evidence of racial discrimination against these women. For other outcomes, the ethnicity/skin color effect disappeared after adjusting for mediators, suggesting mitigation or disappearance of the skin color effect in some practices/interventions in childbirth.
\end{abstract}

Key words Racism, Delivery, Live birth
Resumo O objetivo do presente estudo foi avaliar desigualdade racial na atenção ao parto e ao nascimento na Rede Cegonha utilizando indicadores de boas práticas e intervenções obstétricas. Desigualdade racial, mensurada pelo efeito total da raça/cor no modelo sem ajuste, foi detectada em muitos indicadores. A persistência do efeito direto, após ajuste para os mediadores idade, escolaridade, paridade, hospital de alto risco e região geográfica, sugere discriminação racial contra as pretas, que tiveram menos partograma preenchido ( $R P$ 0,88; IC 95\% 0,80-0,95). Comparadas às brancas, as pretas ficaram menos em litotomia ( $R P$ 0,93; IC95\% 0,89-0,98), realizaram menos episiotomia (RP 0,81; IC95\% 0,68-0,96) e tiveram menos dor na sutura da episiotomia (RP 0,66; IC95\% 0,510,87), sugerindo que boas práticas estariam sendo mais realizadas nas pretas. Entretanto, pelo modelo intervencionista de assistência, ainda adotado por muitos profissionais, essas práticas são de rotina e a menor realização delas nas pretas seria melhor interpretada como evidência de discriminação racial a essas mulheres. Para outros desfechos, o efeito da raça/cor desapareceu após o ajuste para mediadores, sugerindo atenuação ou desaparecimento do efeito da cor da pele em algumas práticas/intervenções na assistência ao parto e nascimento.

Palavras-chave Racismo, Parto, Nascimento vivo 


\section{Introdução}

Desigualdades raciais em vários desfechos de saúde física e mental, bem como no acesso e na utilização de serviços de saúde têm sido bem documentadas ${ }^{1}$. No Brasil, evidências científicas apontam que existe associação entre cor da pele e indicadores da atenção ao pré-natal e parto e mortalidade materna ${ }^{2,3}$. Diversos estudos observaram que a cor da pele preta ou parda esteve associada à inadequação na atenção pré-natal ${ }^{2,4-7}$, à falta de vinculação à maternidade ${ }^{3}$, peregrinação em busca de atendimento médico ${ }^{2,3}$, pior avaliação do tempo de espera para o atendimento médico, diminuição da privacidade ${ }^{8} \mathrm{e}$ falta de acompanhante durante o trabalho de parto, além de menor realização de anestesia local para episiotomia ${ }^{3}$.

No estudo Nascer no Brasil, realizado em 2011/2012, de abrangência nacional, mulheres pardas e sobretudo pretas apresentaram indicadores de atenção ao pré-natal e ao parto menos satisfatórios do que as brancas. Mesmo após controle para variáveis socioeconômicas, demográficas e reprodutivas, a desigualdade racial persistiu para a maioria dos indicadores estudados ${ }^{3}$.

Em 2017, foram avaliadas as boas práticas e as intervenções obstétricas na atenção ao parto e nascimento em maternidades públicas que integram a Rede Cegonha (RC), estratégia do Ministério da Saúde (MS), implantada em 2011. Aproveitando esse cenário, o objetivo do presente estudo foi avaliar a desigualdade racial na assistência ao parto e nascimento por meio de indicadores de boas práticas e intervenções obstétricas, em maternidades da Rede Cegonha. Além disso, verificar se essas desigualdades se reduzem ou desaparecem após ajuste para variáveis sociodemográficas, reprodutivas e de assistência à saúde, seis anos após a realização do estudo Nascer no Brasil, período em que foram desenvolvidas iniciativas governamentais de fortalecimento e fomento dos planos de ação da Rede Cegonha, na perspectiva da assistência humanizada.

\section{Métodos}

Foram utilizados dados primários da pesquisa intitulada "Avaliação da Atenção ao Parto e Nascimento em Maternidades da Rede Cegonha" realizada pela Universidade Federal do Maranhão (UFMA) e pela Escola Nacional de Saúde Pública Sérgio Arouca (ENSP), da Fundação Oswaldo Cruz (Fiocruz), em 2017, sob a coordenação do Ministério da Saúde.
Neste estudo, foram selecionados todos os hospitais públicos e conveniados ao Sistema Único de Saúde do Brasil onde ocorreram partos em 2015, situados em região de saúde com plano de ação da Rede Cegonha aprovados pelo MS. Para fins deste estudo, todos os pontos de atenção ao parto e nascimento serão denominados maternidades, independentemente de serem maternidade ou um setor específico de hospitais gerais, totalizando 606 estabelecimentos?.

A amostra de puérperas foi estratificada por regiões geográficas. O tamanho da amostra foi calculado com base em uma taxa de cesariana de $50 \%$, para detectar diferenças de 5\%, com nível de significância de 0,05 e poder de $80 \%$. Para cada região, foi definido um número fixo de dias de coleta de dados de acordo com o volume de partos nos hospitais elegíveis, sendo de dois dias nas regiões Sudeste e Nordeste, quatro dias na região Norte, cinco dias na região Sul e sete dias na região Centro-Oeste.

Foi aplicado questionário em entrevista face a face com todas as mulheres, e realizada análise de prontuários. Neste artigo foram analisados somente dados de mulheres que tiveram parto vaginal e feto único, nos dias de coleta de dados e que tiveram dados extraídos do prontuário durante a internação, utilizando-se formulários eletrônicos. Do total de 10.665 puérperas entrevistadas, foram analisados os dados de 5.851 mulheres que tiveram gestação única e parto vaginal. Essa amostra representou 55\% do total de mulheres entrevistadas.

\section{Modelo Teórico}

O modelo teórico definido para se mensurar o efeito da raça/cor nas boas práticas de atenção ao parto e nascimento ou nas intervenções obstétricas está demonstrado no gráfico acíclico direcionado (DAG) na Figura $1^{10}$, utilizado para identificar confundimento e mediadores. As variáveis mais importantes para a mensuração do efeito da cor da pele na assistência ao parto e nascimento foram identificadas com base na literatura. Pelo modelo teórico utilizado com base em Blank et al. ${ }^{11}$ e Pearl e Mackenzie ${ }^{12}$, a variável raça/cor foi considerada mais distal, não tendo seu efeito confundido por nenhuma variável antecedente. Como confundimento é um conceito causal, escolaridade e renda não poderiam causar cor da pele. A desigualdade racial foi considerada como o efeito total da raça/cor nas boas práticas/intervenções e estimada pelo coeficiente da raça/cor 


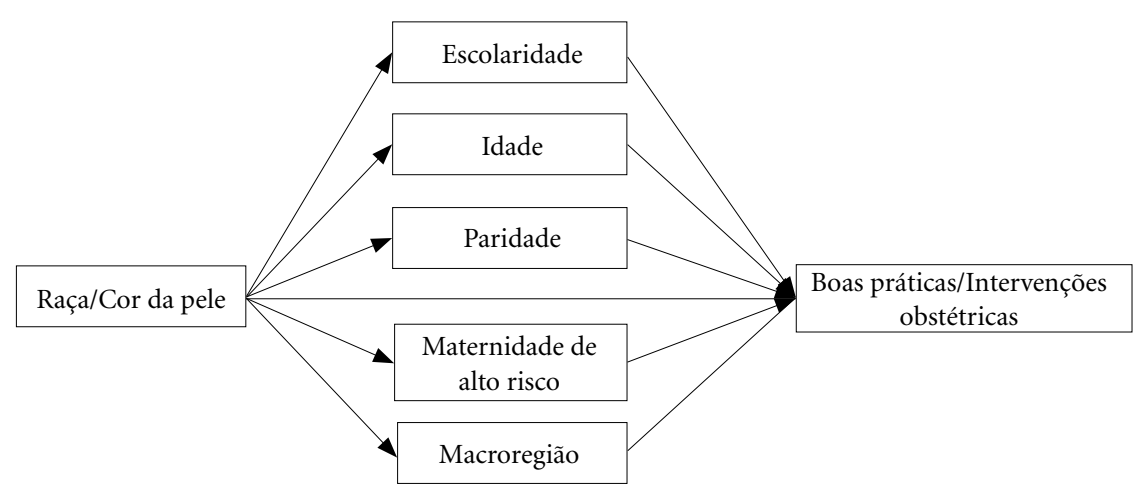

Figura 1. Modelo teórico utilizado para estimativa da desigualdade racial nas boas práticas de atenção ao parto e nascimento e nas intervenções obstétricas.

em modelo de regressão logística não ajustado. O coeficiente correspondente ao efeito direto da variável raça/cor nas boas práticas/intervenções obstétricas foi obtido após ajuste para os seguintes mediadores: escolaridade (superior ou mais, médio completo, fundamental completo, fundamental incompleto), idade em anos ( $<20,20$ a 34 e $\geq 35)$ e paridade (1, 2, 3, 4 ou mais) maternas, macroregião geográfica (Norte, Nordeste, Centro -Oeste, Sudeste e Sul) e ter sido atendida em hospital para gestante de alto risco (sim, não). Esse efeito direto mede o resultado da discriminação racial e de outros mediadores omitidos nas boas práticas/intervenções obstétricas. A persistência do efeito direto após ajuste para os mediadores foi considerada como indicador de discriminação racial/racismo ${ }^{11,12}$.

A variável de exposição foi cor da pele autorreferida, categorizada em branca, preta e parda. Os desfechos foram selecionados a partir da literatura, considerando as práticas avaliadas como promotoras de melhores resultados obstétricos e efetivas para a redução de desfechos negativo ${ }^{13} \mathrm{e}$ que compõem o modelo tecnológico de assistência ao parto e ao nascimento nos serviços da Rede Cegonha ${ }^{14}$. Essas variáveis foram categorizadas em sim ou não e organizadas em três dimensões:

1. Boas práticas na atenção ao trabalho de parto vaginal e outros momentos assistenciais oferta de líquidos ou alimentos durante o trabalho de parto ("Foram oferecidos líquidos, água, sucos, sopas ou algum alimento durante o seu trabalho de parto?"), mobilidade durante o primeiro estágio do trabalho de parto ("Você pôde ficar fora da cama e andar durante o trabalho de parto?"), oferta de métodos não farmacológicos para alívio da dor ("Foi ofertado algumas das medidas?"), preenchimento do partograma no prontuário (extração de dados do prontuário), presença de acompanhante durante todo o período de hospitalização ("A maternidade permitiu que o seu acompanhante ficasse com você o tempo todo?"), e se sentir poucas vezes/raramente bem tratada ("Com que frequência você está se sentindo acolhida, bem tratada e respeitada durante sua internação, nessa maternidade?").

2. Intervenções no trabalho de parto e no parto vaginal - uso de cateter venoso ("Quando você estava no trabalho de parto foi colocado soro na veia?"), amniotomia para mulheres com bolsa íntegra na admissão ("Romperam a sua bolsa depois que você chegou no hospital?"), analgesia raque/epidural ("Você recebeu medicação/ analgesia para alívio da dor durante o trabalho de parto?"), uso de ocitocina (extração de dados do prontuário ), litotomia ("Qual foi a posição que você ficou para ter o bebê?"), episiotomia (“Cortaram o seu períneo (vagina) no parto?”) e dor na sutura da episiotomia ("Você sentiu dor no momento de suturar (costurar, reparar) o períneo?"); manobra de Kristeller (“Na hora do parto, alguém apertou/subiu na sua barriga para ajudar a saída do bebê?"), e;

3. Boas práticas na atenção ao recém-nascido - contato pele a pele ("Imediatamente após o nascimento, ainda no local de parto, o seu bebê foi colocado em contato com a sua pele, sem nenhuma roupa ou lençol entre vocês?"), amamen- 
tar no local do parto ("Depois do nascimento, você deu o peito no local de parto?”), amamentar nas primeiras 24 horas de vida do recém-nascido ("Você amamentou o seu bebe nas primeiras 24 horas após o parto?") e oferta de formula láctea (“O bebê tomou outro leite que não o seu?”).

\section{Análise estatística}

As variáveis em estudo foram descritas por meio de frequências absoluta e relativa. Para avaliar o efeito total da variável de exposição raça/cor da pele para cada desfecho de interesse foi utilizado modelo de regressão de Poisson simples. A desigualdade racial foi detectada pelo efeito total, obtido no modelo sem ajuste. No modelo de regressão de Poisson múltipla foram considerados no ajuste os mediadores descritos no modelo teórico, com o objetivo de estimar o efeito direto da cor da pele para cada uma das boas práticas/intervenções empregadas na assistência ao parto e nascimento. Para compensar as probabilidades desiguais de seleção das puérperas foram incluídos nas análises os pesos de ponderação, determinados pelo inverso da probabilidade de seleção de cada mulher. Em todas as análises, realizadas no STATA 14.0, foram estimadas as razões de prevalência (RP) e respectivos intervalos de confiança (IC) de 95\%, tendo em vista que os desfechos estudados apresentavam prevalências maiores que $10 \%{ }^{15}$. Os erros padrão foram estimados por método robusto.

O projeto seguiu as recomendações da Resolução CNS 466/2012 e foi aprovado pelo CEP da Universidade Federal do Maranhão e da Escola Nacional de Saúde Pública Sérgio Arouca. Todas as mulheres assinaram o Termo de Consentimento Livre e Esclarecido.

\section{Resultados}

Um percentual de $58,7 \%$ se identificou com cor de pele parda, $28,0 \%$ branca e $13,3 \%$ preta. A maioria tinha entre 20 e 34 anos de idade $(68,3 \%)$, dois ou mais filhos $(52,0 \%)$, escolaridade até nível médio incompleto $(58,4 \%)$ e parto ocorrido em hospitais para atendimento de gravidez de risco habitual $(69,5 \%)$ (Tabela 1$)$.

$\mathrm{Na}$ dimensão boas práticas no trabalho de parto e no parto, as mulheres de cor de pele parda, quando comparadas com as de cor branca, na análise bivariada, tiveram menor oferta de líquidos ou outros alimentos durante o trabalho de parto (RP 0,91 IC95\% 0,84-0,98), menor oferta de alívio não farmacológico da dor ( $\mathrm{RP}$ 0,88, IC95\% 0,84-0,93), menor preenchimento do partograma (RP 0,85 IC95\% 0,80-0,90) e em maior percentual foram poucas vezes bem tratadas durante a internação (RP 1,38 IC95\% 1,10$1,74)$. As mulheres de cor de pele preta tiveram menor oferta de medidas não farmacológicas para alivio da dor (RP 0,90 IC95\% 0,83-0,97), assim como menor preenchimento do partograma (RP 0,82 IC95\% 0,75-0,90). Ainda na análise bivariada, não houve diferença entre cor da pele com permissão para deambular durante o trabalho de parto e ter acompanhante durante todo o tempo (Tabela 2).

Nas intervenções no trabalho de parto e no parto vaginal, na análise bivariada, mulheres de cor de pele parda tiveram menor realização de venóclise (RP 0,93 IC95\% 0,86-0,99), analgesia (RP 0,77 IC95\% 0,64-0,92), uso de ocitocina (RP 0,90 IC95\% 0,81-0,99) e episiotomia (RP 0,86 IC95\% 0,76-0,97). As mulheres de cor de pele preta tiveram menores percentuais de parto em posição outra que não a litotomia (RP 0,94 IC95\% 0,90-0,98) e de ter sido feita episiotomia (RP 0,82 IC95\% 0,69-0,97), assim como de referir dor durante a sutura da episiotomia (RP 0,74 IC95\% 0,57-0,96), tendo como referência as mulheres de cor branca. As manobras de amniotomia após a internação e Kristeller na hora do parto, na análise bivariada, não mostraram diferença estatisticamente significante segundo a cor da pele (Tabela 3 ).

Em relação às boas práticas com o recémnascido, na análise bivariada, ter cor de pele preta foi associada a maior realização de contato pele a pele (RP 1,08 IC95\% 1,01-1,16) e a menor percentual de amamentação nas primeiras 24 horas (RP 0,94 IC95\% 0,89-0,99) (Tabela 4).

Após o ajuste, na dimensão de boas práticas, somente menor preenchimento do partograma (RP 0,88 IC95\% 0,80-0,95) se manteve associado à cor de pele preta (Tabela 2). Nas intervenções no trabalho de parto e no parto, mulheres de cor de pele preta realizaram menos litotomia (RP 0,93 IC95\% 0,89-0,98), episiotomia (RP 0,81 IC95\% 0,68-0,96) e tiveram menos dor na sutura da episiotomia (RP 0,66 IC95\% 0,51-0,87). Mulheres de cor de pele parda referiram mais dor durante a sutura da episiotomia do que as mulheres de cor de pele branca (RP 0,84 IC95\% 0,71-0,99) (Tabela 3). Maior realização de contato pele a pele com o recém-nascido foi observado nas mulheres de cor de pele preta (RP 1,09 IC95\% 1,02-1,17) ou parda (RP 1,06 IC95\% 1,01-1,12), comparadas às brancas. Mulheres de cor de pele preta também 
Tabela 1. Características das puérperas de parto vaginal único em maternidades da Rede Cegonha. Brasil, 2017.

\begin{tabular}{|c|c|c|}
\hline Variáveis & $\mathbf{n}$ & $\begin{array}{c}\% \\
\text { ponderado }\end{array}$ \\
\hline \multicolumn{3}{|l|}{ Cor da pele } \\
\hline Branca & 1.785 & 28,0 \\
\hline Preta & 718 & 13,3 \\
\hline Parda & 3.299 & 58,7 \\
\hline \multicolumn{3}{|l|}{ Idade em anos } \\
\hline Até 19 & 1.331 & 23,1 \\
\hline 20 a 34 & 3.949 & 68,3 \\
\hline$\geq 35$ & 506 & 8,5 \\
\hline \multicolumn{3}{|l|}{ Escolaridade } \\
\hline Superior ou mais & 295 & 4,9 \\
\hline Médio completo & 2.113 & 36,7 \\
\hline Fundamental completo & 1.753 & 29,7 \\
\hline $\begin{array}{l}\text { Fundamental } \\
\text { incompleto }\end{array}$ & 1.673 & 28,7 \\
\hline \multicolumn{3}{|l|}{ Paridade } \\
\hline 1 & 2.651 & 48,0 \\
\hline 2 & 1.577 & 25,9 \\
\hline 3 & 832 & 13,2 \\
\hline 4 ou mais & 770 & 12,9 \\
\hline \multicolumn{3}{|l|}{$\begin{array}{l}\text { Hospital para gestantes de } \\
\text { alto risco }\end{array}$} \\
\hline Não & 4.263 & 69,5 \\
\hline Sim & 1.573 & 30,5 \\
\hline \multicolumn{3}{|l|}{ Macroregião geográfica } \\
\hline Norte & 1.036 & 13,0 \\
\hline Nordeste & 1.170 & 30,7 \\
\hline Centro Oeste & 998 & 7,3 \\
\hline Sudeste & 1.460 & 37,7 \\
\hline Sul & 1.187 & 11,3 \\
\hline
\end{tabular}

amamentaram mais seus filhos no local do parto (RP 1,14 IC95\% 1,01-1,30) do que as brancas (Tabela 4).

\section{Discussão}

Foram analisados 18 indicadores selecionados de boas práticas e intervenções obstétricas na assistência ao parto e ao nascimento em maternidades da Rede Cegonha, sendo que foi observada desigualdade racial em 12 deles (alimentação na primeira fase do trabalho de parto, alivio não farmacológico da dor, partograma preenchido, poucas vezes bem tratada, venóclise, analgesia, uso de citocina, litotomia, episiotomia, dor na sutura da episiotomia, contato pele a pele ao nascimento e amamentar nas primeiras 24 horas). Entretanto, após ajuste para mediadores a associação persistiu para seis indicadores (partograma preenchido, litotomia, episiotomia, dor na sutura da episiotomia, contato pele a pele e amamentar no local do parto), sugerindo atenuação do efeito da cor da pele nas práticas/intervenções utilizadas na assistência ao parto e nascimento, para alguns indicadores. Algumas práticas foram universalizadas, como a presença de acompanhante, não existindo diferenças entre brancas e pretas/pardas.

Efeito direto da cor da pele nos desfechos estudados significante após o ajuste para mediadores sugere a existência de discriminação racial/ racismo nessas práticas assistenciais nos serviços de atendimento ao parto e nascimento. Por outro lado, o desaparecimento do efeito da raça/cor após o ajuste para os mediadores em alguns dos desfechos analisados sugere que esses mediadores explicaram o efeito da desigualdade racial nesses casos. Essas conclusões se aplicam em expectativa, assumindo-se que o nosso modelo teórico esteja correto e que todos os mediadores da raça/cor nos desfechos estudados tenham sido incluídos no ajuste. A grande vantagem do DAG é deixar os pressupostos da análise explícitos. Entretanto, nunca é possível saber se todas as variáveis importantes foram incluídas, se o diagrama é correto, se não há confundimento por variável omitida.

Pretas e/ou pardas tiveram menor oferta de líquidos ou outros alimentos durante o trabalho de parto, menor oferta de métodos de alívio não farmacológico da dor, foram bem tratadas poucas vezes, foram menos submetidas a intervenções como venóclise, analgesia e uso de ocitocina comparadas com as brancas. A menor ocorrência dessas boas práticas ou intervenções nas pretas e/ou pardas foram explicadas pelos mediadores utilizados no ajuste, o que torna improvável que a desigualdade racial na assistência ao parto e nascimento detectada nessas variáveis seja reflexo da discriminação racial no âmbito dos serviços de saúde.

Para seis indicadores de boas práticas e intervenções obstétricas não foram detectadas diferenças segundo a cor da pele no modelo bruto. Dessa forma, não foi constatada desigualdade racial na mobilidade durante o primeiro estágio do trabalho de parto, presença de acompanhante durante todo o tempo de internação, realização de amniotomia e manobra de Kristeller, amamentação no local do parto e oferta de fórmula láctea para o bebê. Esse achado aponta para a 
Tabela 2. Boas práticas no trabalho de parto e em outros momentos assistenciais segundo cor da pele autorreferida em maternidades da Rede Cegonha. Brasil, 2017.

\begin{tabular}{|c|c|c|c|c|c|c|c|c|}
\hline & \multirow[b]{2}{*}{$\mathbf{n}$} & \multirow[b]{2}{*}{$\%$} & \multicolumn{3}{|c|}{ Bruta $^{*}$} & \multicolumn{3}{|c|}{ Ajustada $^{* *}$} \\
\hline & & & RP & IC95\% & P valor & RP & IC95\% & $P$ valor \\
\hline \multicolumn{9}{|c|}{ Alimentação } \\
\hline Branca & 1706 & 49,6 & 1 & - & - & 1 & - & - \\
\hline Preta & 676 & 48,5 & 0,98 & $0,87-1,09$ & 0,701 & 1,01 & $0,90-1,14$ & 0,772 \\
\hline Parda & 3084 & 45,3 & 0,91 & $0,84-0,98$ & 0,023 & 0,97 & $0,90-1,06$ & 0,597 \\
\hline \multicolumn{9}{|c|}{ Movimentação } \\
\hline Branca & 1650 & 92,4 & 1 & - & - & 1 & - & - \\
\hline Preta & 653 & 90,6 & 0,98 & $0,94-1,01$ & 0,246 & 0,98 & $0,94-1,01$ & 0,342 \\
\hline Parda & 2992 & 91,1 & 0,98 & $0,96-1,01$ & 0,171 & 0,98 & $0,96-1,01$ & 0,334 \\
\hline \multicolumn{9}{|c|}{ Oferta de alívio não farmacológico } \\
\hline Branca & 1785 & 72,3 & 1 & - & - & 1 & - & - \\
\hline Preta & 718 & 65,4 & 0,90 & $0,83-0,97$ & 0,009 & 0,94 & $0,87-1,02$ & 0,171 \\
\hline Parda & 3299 & 64,3 & 0,88 & $0,84-0,93$ & $<0,001$ & 0,95 & $0,90-1,01$ & 0,121 \\
\hline \multicolumn{9}{|c|}{ Preenchimento do partograma } \\
\hline Branca & 1734 & 70,7 & 1 & - & & 1 & - & \\
\hline Preta & 674 & 58,4 & 0,82 & $0,75-0,90$ & $<0,001$ & 0,88 & $0,80-0,95$ & 0,004 \\
\hline Parda & 3140 & 60,3 & 0,85 & $0,80-0,90$ & $<0,001$ & 0,96 & $0,90-1,01$ & 0,172 \\
\hline \multicolumn{9}{|c|}{ Acompanhante permitido todo tempo } \\
\hline Branca & 1592 & 88,5 & 1 & - & - & 1 & - & - \\
\hline Preta & 619 & 89,0 & 1,00 & $0,96-1,04$ & 0,790 & 1,01 & $0,97-1,05$ & 0,642 \\
\hline Parda & 2860 & 86,6 & 0,97 & $0,94-1,01$ & 0,172 & 0,98 & $0,95-1,01$ & 0,262 \\
\hline \multicolumn{9}{|c|}{ Poucas vezes foi bem tratada } \\
\hline Branca & 1779 & 7,6 & 1 & - & - & 1 & - & - \\
\hline Preta & 716 & 8,6 & 1,13 & $0,81-1,58$ & 0,453 & 0,93 & $0,66-1,31$ & 0,694 \\
\hline Parda & 3285 & 10,6 & 1,38 & $1,10-1,74$ & 0,005 & 1,04 & $0,82-1,32$ & 0,725 \\
\hline
\end{tabular}

* Razões de prevalências (RP) e Intervalo de confiança (IC) de 95\% obtidas em modelo de regressão de Poisson simples com estimativa robusta da variância e ponderado pelo inverso da probabilidade de seleção de cada puérpera. ${ }^{\star \star}$ Estimativas obtidas em modelo de regressão de Poisson múltipla ajustado por idade, escolaridade, paridade, hospital de alto risco e macroregião geográfica com estimativa robusta da variância e ponderado pelo inverso da probabilidade de seleção de cada puérpera.

existência de possível atenuação do efeito da cor da pele em práticas anteriormente hegemônicas na atenção ao parto hospitalar. Essa evidência fala a favor de um modelo de atenção ao parto e nascimento em transformação, na tentativa de redução das desigualdades raciais.

Estudos apontam que a presença do acompanhante durante a assistência ao parto tem sido capaz de promover mudanças nas condutas profissionais, tornando-as mais humanas e menos rotineiras. A mudança estrutural dessa e de outras práticas específicas pode ter corrigido possíveis desigualdades ${ }^{16}$.

Para seis indicadores o efeito direto da raça nas boas práticas e intervenções obstétricas no trabalho de parto e parto foi significante mesmo após ajuste para mediadores. Mulheres pretas tiveram menor proporção de partograma preenchido, foram colocadas menos vezes em posição de litotomia, foram menos submetidas à episiotomia e referiram menos dor durante a sutura da episiotomia. Além disso, realizaram mais contato pele a pele com seus bebês e amamentaram mais no local do parto quando comparadas às brancas. Para as mulheres pardas dois indicadores foram significantes após o ajuste para mediadores. Essas mulheres relataram menos dor na sutura da episiotomia e realizaram mais contato pele a pele com o recém-nascido. Como dito acima, a persistência da desigualdade racial nesses seis indicadores, após o ajuste para mediadores, sugere que a discriminação racial/racismo está presente na atenção ao parto e nascimento. Desse modo, pardas e, especialmente, pretas são tratadas de forma diferente em muitos serviços de saúde apenas porque possuem pele de cor diferente e não devido a outras diferenças socioeconômicas, demográficas, regionais ou relativas a serviços de saúde. 
Tabela 3. Intervenções no Trabalho de Parto e no Parto Vaginal segundo cor da pele autorrreferida em maternidades da Rede Cegonha. Brasil, 2017.

\begin{tabular}{|c|c|c|c|c|c|c|c|c|}
\hline & \multirow[b]{2}{*}{$\mathbf{n}$} & \multirow[b]{2}{*}{$\%$} & \multicolumn{3}{|c|}{ Bruta $^{*}$} & \multicolumn{3}{|c|}{ Ajustada $^{\star \star}$} \\
\hline & & & RP & IC & pvalor & $\mathbf{R P}$ & IC & pvalor \\
\hline \multicolumn{9}{|l|}{ Venóclise } \\
\hline Branca & 1719 & 59,0 & 1 & - & - & 1 & - & - \\
\hline Preta & 690 & 57,1 & 0,96 & $0,87-1,06$ & 0,495 & 0,99 & $0,90-1,09$ & 0,881 \\
\hline Parda & 3134 & 55,0 & 0,93 & $0,86-0,99$ & 0,042 & 0,99 & $0,92-1,06$ & 0,811 \\
\hline \multicolumn{9}{|l|}{ Analgesia } \\
\hline Branca & 1711 & 19,2 & 1 & - & - & 1 & - & - \\
\hline Preta & 685 & 18,0 & 0,93 & $0,73-1,19$ & 0,596 & 1,02 & $0,79-1,31$ & 0,845 \\
\hline Parda & 3127 & 14,9 & 0,77 & $0,64-0,92$ & 0,006 & 0,91 & $0,75-1,10$ & 0,343 \\
\hline \multicolumn{9}{|c|}{ Amniotomia } \\
\hline Branca & 1690 & 44,3 & 1 & - & - & 1 & - & - \\
\hline Preta & 679 & 41,5 & 0,93 & $0,82-1,06$ & 0,304 & 0,95 & $0,83-1,08$ & 0,439 \\
\hline Parda & 3102 & 41,7 & 0,94 & $0,86-1,02$ & 0,167 & 0,96 & $0,88-1,06$ & 0,528 \\
\hline \multicolumn{9}{|c|}{ Uso de ocitocina } \\
\hline Branca & 1768 & 39,6 & 1 & - & - & 1 & - & - \\
\hline Preta & 704 & 39,7 & 1,00 & $0,87-1,15$ & 0,966 & 1,08 & $0,95-1,24$ & 0,210 \\
\hline Parda & 3257 & 35,7 & 0,90 & $0,81-0,99$ & 0,034 & 1,01 & $0,91-1,11$ & 0,818 \\
\hline \multicolumn{9}{|l|}{ Litotomia } \\
\hline Branca & 1779 & 88,2 & 1 & - & - & 1 & - & - \\
\hline Preta & 716 & 83,3 & 0,94 & $0,90-0,98$ & 0,017 & 0,93 & $0,89-0,98$ & 0,008 \\
\hline Parda & 3288 & 87,4 & 0,99 & $0,96-1,01$ & 0,511 & 0,97 & $0,94-1,01$ & 0,120 \\
\hline \multicolumn{9}{|c|}{ Episiotomia } \\
\hline Branca & 1706 & 31,5 & 1 & - & - & 1 & - & - \\
\hline Preta & 678 & 25,9 & 0,82 & $0,69-0,97$ & 0,024 & 0,81 & $0,68-0,96$ & 0,016 \\
\hline Parda & 3136 & 27,3 & 0,86 & $0,76-0,97$ & 0,018 & 0,89 & $0,79-1,00$ & 0,057 \\
\hline \multicolumn{9}{|c|}{ Dor na sutura da episiotomia } \\
\hline Branca & 497 & 42,7 & 1 & - & - & 1 & - & - \\
\hline Preta & 172 & 31,7 & 0,74 & $0,57-0,96$ & 0,024 & 0,66 & $0,51-0,87$ & 0,003 \\
\hline Parda & 862 & 39,8 & 0,93 & $0,80-1,09$ & 0,382 & 0,84 & $0,71-0,99$ & 0,041 \\
\hline \multicolumn{9}{|c|}{ Manobra de Kristeller } \\
\hline Branca & 1738 & 15,7 & 1 & - & - & 1 & - & - \\
\hline Preta & 696 & 16,9 & 1,07 & $0,83-1,38$ & 0,567 & 1,04 & $0,80-1,35$ & 0,725 \\
\hline Parda & 3188 & 15,7 & 0,99 & $0,83-1,18$ & 0,970 & 0,96 & $0,80-1,15$ & 0,693 \\
\hline
\end{tabular}

${ }^{*}$ Razões de prevalências (RP) e Intervalo de confiança (IC) de $95 \%$ obtidas em modelo de regressão de Poisson simples com estimativa robusta da variância e ponderado pelo inverso da probabilidade de seleção de cada puérpera. ${ }^{* *}$ Estimativas obtidas em modelo de regressão de Poisson múltipla ajustado c com estimativa robusta da variância e ponderado pelo inverso da probabilidade de seleção de cada puérpera.

Observou-se que na assistência ao parto de mulheres pretas houve menor preenchimento do partograma, apontando para discriminação racial contra essas mulheres. Entretanto, há outras intervenções, como litotomia e episiotomia, que foram realizadas em menor proporção nas pretas. Como as boas práticas consideram que essas intervenções não devem ser feitas de rotina, de acordo com as normas do $\mathrm{MS}^{17}$, sua menor realização nas mulheres pretas poderia estar indicando um benefício ou privilégio a essas mulheres, uma discriminação "às avessas”, com as brancas recebendo pior tratamento. Entretanto, tais práticas são consideradas "boas" no modelo biomédico intervencionista de assistência ao parto, ainda prevalente na prática de muitos profissionais ${ }^{18}$. Por exemplo, no entendimento de alguns profissionais, o correto seria realizar a episiotomia. Portanto, quando alguns profissionais negam essa intervenção às mulheres pretas, essa negação seria melhor interpretada como evidência de discriminação racial, muito embora essa 
Tabela 4. Boas práticas na atenção ao recém-nascido segundo cor da pele autorreferida em maternidades da Rede Cegonha. Brasil, 2017.

\begin{tabular}{|c|c|c|c|c|c|c|c|c|}
\hline & \multirow[b]{2}{*}{$\mathbf{n}$} & \multirow[b]{2}{*}{$\%$} & \multicolumn{3}{|c|}{ Bruta $^{*}$} & \multicolumn{3}{|c|}{ Ajustada $^{\star \star}$} \\
\hline & & & RP & IC95\% & pvalor & RP & IC95\% & pvalor \\
\hline \multicolumn{9}{|c|}{ Contato pele a pele } \\
\hline Branca & 1749 & 66,5 & 1 & - & - & 1 & - & - \\
\hline Preta & 695 & 72,0 & 1,08 & $1,01-1,16$ & 0,023 & 1,09 & $1,02-1,17$ & 0,010 \\
\hline Parda & 3196 & 69,6 & 1,04 & $0,99-1,10$ & 0,088 & 1,06 & $1,01-1,12$ & 0,027 \\
\hline \multicolumn{9}{|c|}{ Amamentar no local do parto } \\
\hline Branca & 1744 & 40,8 & 1 & - & - & 1 & - & - \\
\hline Preta & 695 & 44,5 & 1,09 & $0,96-1,23$ & 0,173 & 1,14 & $1,01-1,30$ & 0,029 \\
\hline Parda & 3201 & 40,3 & 0,98 & $0,89-1,08$ & 0,800 & 1,04 & $0,94-1,14$ & 0,413 \\
\hline \multicolumn{9}{|c|}{ Amamentar nas primeiras $24 \mathrm{~h}$} \\
\hline Branca & 992 & 89,7 & 1 & - & - & 1 & - & - \\
\hline Preta & 388 & 84,7 & 0,94 & $0,89-0,99$ & 0,044 & 0,95 & $0,89-1,01$ & 0,097 \\
\hline Parda & 1875 & 89,8 & 1,00 & $0,96-1,03$ & 0,942 & 1,00 & $0,96-1,03$ & 0,836 \\
\hline \multicolumn{9}{|c|}{ Oferta de fórmula láctea } \\
\hline Branca & 1698 & 16,5 & 1 & - & - & 1 & - & - \\
\hline Preta & 669 & 16,4 & 0,99 & $0,78-1,26$ & 0,979 & 1,02 & $0,79-1,30$ & 0,876 \\
\hline Parda & 3098 & 14,0 & 0,84 & $0,70-1,01$ & 0,072 & 0,92 & $0,76-1,12$ & 0,455 \\
\hline
\end{tabular}

negação, nesse caso, termine sendo benéfica para essas mulheres.

Para indicadores do recém-nascido, mulheres pretas amamentaram mais no local do parto e tiveram mais contato pele a pele com seus bebês. A interpretação nesse caso é que as pretas e pardas estão realizando essas boas práticas relacionadas ao recém-nascido em maior proporção do que as brancas. Isso pode ser reflexo da maior aceitação dessas práticas por mulheres negras.

$\mathrm{Na}$ pesquisa Nascer no Brasil, realizada em 2011/2012 a desigualdade racial persistiu para a maioria dos indicadores analisados após o controle de mediadores ${ }^{3}$. No presente estudo, realizado seis anos depois, em 2017, a desigualdade racial desapareceu para a maioria das variáveis estudadas após o ajuste, o que pode indicar uma tendência de redução da discriminação racial nos serviços de saúde. Entretanto, pelas diferenças nas duas amostras essa possibilidade deve ser interpretada com cautela. No Nascer no Brasil a amostra foi composta por partos vaginais e cesáreos ocorridos em instituições públicas, privadas e mistas (maternidades privadas conveniadas ao SUS) com 500 ou mais partos em 2007, enquanto na RC foram incluídos apenas partos vaginais realizados em instituições públicas e mistas, com mais de 500 partos ocorridos em 2015, localizadas em região de saúde com plano de ação da RC aprovados pelo MS. Ou seja, a amostra do presente estudo foi composta de hospitais públicos onde os programas de humanização do parto e nascimento ${ }^{19}$ provavelmente estavam implantados há mais tempo e havia maior motivação e desejo de mudança nas práticas assistenciais.

A noção de que os seres humanos merecem respeito pelo ideal de humanidade e dignidade como princípio dos Direitos Humanos, pode explicar esse avanço, na medida em que tem guiado o enfrentamento de todas as formas de discriminação e injustiças sociais. No plano da teoria política, o princípio de igualdade e o princípio de tolerância estão na base da Declaração Universal dos Direitos Humanos ${ }^{20}$. Nesse sentido supõe-se que a sociedade tenha se tornado mais tolerante, com melhor aceitação do desigual, na época da realização do campo deste estudo ${ }^{21}$. Além disso, nos últimos anos, tem-se observado esforço do MS para a promoção e a qualificação da atenção ao parto com valorização das diretrizes da Política Nacional de Humanização ${ }^{22,23}$. Esses esforços se deram principalmente pela criação e imple- 
mentação da estratégia RC, pela divulgação de manuais técnicos e ações de qualificação dos profissionais de saúde $\mathrm{e}^{14,22,24}$.

Conformando uma limitação do estudo, apontamos que os indicadores socioeconômicos que foram utilizados como mediadores podem não ser marcadores adequados das circunstâncias sociais. Pondera-se que a mediação socioeconômica não responde integralmente pelas diferenças de saúde entre os grupos sociais. As divisões raciais podem gerar efeitos adicionais na saúde que não dependem das diferenças socioeconômicas entre as pessoas. Não é possível afirmar que a discriminação racial possa ser totalmente explicada pelas variáveis do modelo proposto. Porém, potencialmente afetam as relações de interesse neste estudo por refletirem, em grande parte, as diferenças existentes nos níveis sociais e econômicos no Brasil. Além disso, como discutido por Santos ${ }^{25}$, além dos efeitos indiretos na saúde, por meio da posição socioeconômica, as exposições à discriminação e ao racismo podem também afetar diretamente a saúde através do ambiente social e do acúmulo de adversidades em múltiplos domínios e aspectos, tais como viés racial no serviço de saúde e do estresse advindo de experiências de discriminação. Outra limita- ção da abordagem utilizada é que a cor/raça pode ter efeitos indiretos nas boas práticas e intervenções obstétricas no parto e nascimento que não foram calculados neste estudo.

Ressaltamos que os dados aqui discutidos dizem respeito às maternidades integrantes da $\mathrm{RC}$ nas cinco regiões brasileiras. Essas maternidades são públicas ou privadas conveniadas com o SUS e de porte variado e juntas respondem por mais

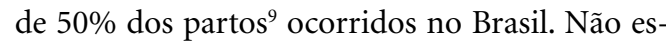
tão incluídos dados provenientes de hospitais/ maternidades que atendem exclusivamente ao setor privado, não conveniadas ao SUS. O estudo avalia a estratégia $\mathrm{RC}$ e possíveis atenuações de desigualdades da assistência ao parto e nascimento promovidos por esta estratégia.

Os achados apresentados neste estudo apontam para a necessidade de investimentos nos processos de qualificação dos profissionais de saúde com a adoção de políticas de saúde para orientar práticas que visem à redução da desigualdade racial. Também é importante a realização sistemática de monitoramento e avaliação dos indicadores da RC em cada maternidade, com vistas ao aprimoramento da gestão da informação e do cuidado às mulheres e crianças com enfoque na redução da desigualdade e discriminação raciais.

\section{Colaboradores}

Todos os autores contribuíram na preparação deste manuscrito. MTSSB Alves - Análise formal, metodologia, escrita do rascunho original, redação e edição final; DC Chagas - Manejo de software, escrita do rascunho original, redação e edição final; AM Santos - Análise, metodologia e escrita do rascunho original; VMF Simões e BVS Ayres - Metodologia e redação e edição final; GL Santos - Administração do projeto e escrita do rascunho original; AAM Silva - Concepção, análise, metodologia e redação e edição final. 


\section{Referências}

1. Hicken MT, Kravitz-Wirtz N, Durkee M, Jackson JS. Racial inequalities in health: Framing future research. Soc Sci Med 2018; 199:11-18.

2. Leal MC, Gama SGN, Cunha CB. Desigualdades raciais, sociodemográficas e na assistência ao pré-natal e ao parto, 1999-2001. Rev Saude Publica 2005; 39(1):100-107.

3. Teixeira NZF, Pereira WR, Barbosa DA, Vianna LAC Mortalidade materna e sua interface com a raça em Mato Grosso. Rev Bras Saúde Matern Infant 2012; 12(1):27-35.

4. Leal MC, Gama SGN, Pereira APE, Pacheco VE, Carmo CN, Santos RV. A cor da dor: iniquidades raciais na atenção pré-natal e ao parto no Brasil. Cad Saude Publica 2017; 33(Supl. 1):1-17.

5. Fonseca SC, Kale PL, Silva KS. Pré-natal em mulheres usuárias do Sistema Único de Saúde em duas maternidades no Estado do Rio de Janeiro, Brasil: a cor importa? Rev Bras Saúde Matern Infant 2015; 15(2):209216.

6. Fonseca SC, Monteiro DSA, Pereira CMSC, Scoralick ACD, Jorge MG, Rozario S. Desigualdades no pré-natal em cidade do Sudeste do Brasil. Cien Saude Colet 2014; 19(7):1991-1998.

7. Domingues RMSM, Viellas EF, Dias MAB, Torres JA, Theme-Filha MM, Gama SGN, Leal MC. Adequação da assistência pré-natal segundo as características maternas no Brasil. Rev Panam Salud Pública 2015; 37(3):140-147.

8. d'Orsi E, Brüggemann OM, Diniz CSG, Aguiar JM, Gusman CR, Torres JA, Angulo-Tuesta A, Rattner D, Domingues RMSM. Desigualdades sociais e satisfação das mulheres com o atendimento ao parto no Brasil: estudo nacional de base hospitalar. Cad Saude Publica 2014; 30(Supl. 1):S154-S168.

9. Vilela MEA, Leal MC, Thomaz EBAF, Gomes MASM, Bittencourt DAS, Gama SGN, Silva LBRAA LZ. Avaliação da atenção ao parto e nascimento nas maternidades da Rede Cegonha: os caminhos metodológicos. Cien Saude Colet 2020; 26(3):789-800.

10. Fleischer NL, Roux AVD. Using directed acyclic graphs to guide analyses of neighbourhood health effects: an introduction. J Epidemiol Community Health 2008; 62(9):842-846.

11. Blank RM, Dabady M, Citro CF. Measuring Racial Discrimination. Washington: The National Academic Press; 2004. (Vol 35).

12. Pearl J, Mackenzie D. The Book of Why: The New Science of Cause and Effect. New York: Basic Books; 2018.

13. Leal MC, Pereira APE, Domingues RMSM, Dias MAB, Nakamura-Pereira M, Bastos MH, Gama SGN. Intervenções obstétricas durante o trabalho de parto e parto em mulheres brasileiras de risco habitual. Cad Saude Publica 2014; 30(Supl. 1):S17-S32.
14. Brasil. Portaria de consolidação $\mathrm{n}^{\circ} 3$, de 28 de setembro de 2017. Consolidação das normas sobre as redes do Sistema Único de Saúde. Diário Oficial da União 2017; 28 set.

15. Coutinho L, Scazufca M, Menezes PR. Métodos para estimar razão de prevalência em estudos de corte transversal. Rev Saude Publica 2008; 42(6):992-998.

16. Gomes IEM, Mello Padoin SM, Langendorf TF, Paula CC, Gomes CA, Ribeiro AC. Benefícios da presença do acompanhante no processo de parto e nascimento: revisão integrativa. Rev Enferm da UFSM 2019; 9:61.

17. Comissão Nacional de Incorporação de Tecnologias no SUS (Conitec). Diretriz Nacional de Assistência ao Parto Normal: relatório de recomendação. Brasília: Conitec; 2016.

18. Melo BM, Gomes LFS, Henriques ACPT, Lima SKM, Damasceno AKC. Implementação das boas práticas na atenção ao parto em maternidade de referência. Rev da Rede Enferm do Nord 2017; 18(3):376-382.

19. Andrade MAC, Lima J. O modelo obstétrico e neonatal que defendemos e com o qual trabalhamos. Humaniza do parto e do nascimento. Brasília: MS; 2014.

20. Assembly UNG. Universal declaration of human rights. UN Gen Assem 1948; 302(2).

21. Silva SG. Direitos humanos: entre o princípio de igualdade e a tolerância. Praia Vermelha 2009; 19(1).

22. Brasil. Ministério da Saúde (MS). Pré-natal e puerpério: atenção qualificada e humanizada. Brasília: MS; 2006.

23. Brasil. Ministério da Saúde (MS). HumanizaSUS: documento base para gestores e trabalhadores do SUS. Brasília: MS; 2008.

24. Brasil. Ministério da Saúde (MS). Atenção ao Pré-Natal de Baixo Risco. Brasília: MS; 2012.

25. Santos JAF. Desigualdade racial de saúde e contexto de classe no Brasil. Dados-Revista de Ciências Sociais 2011; 54(1):5-40.

Artigo apresentado em 22/04/2020

Aprovado em 26/10/2020

Versão final apresentada em 28/10/2020

Editores-chefes: Romeu Gomes, Antônio Augusto Moura da Silva 